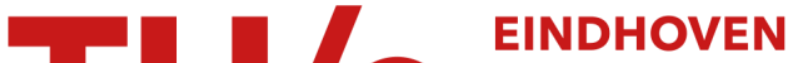

\section{Vibrational dephasing mechanisms in hydrogen-bonded systems}

\section{Citation for published version (APA):}

Bonn, M., Brugmans, M. J. P., Kleyn, A. W., Santen, van, R. A., \& Bakker, H. J. (1996). Vibrational dephasing mechanisms in hydrogen-bonded systems. Physical Review Letters, 76(14), 2440-2443.

https://doi.org/10.1103/PhysRevLett.76.2440

DOI:

10.1103/PhysRevLett.76.2440

Document status and date:

Published: 01/01/1996

\section{Document Version:}

Publisher's PDF, also known as Version of Record (includes final page, issue and volume numbers)

\section{Please check the document version of this publication:}

- A submitted manuscript is the version of the article upon submission and before peer-review. There can be important differences between the submitted version and the official published version of record. People interested in the research are advised to contact the author for the final version of the publication, or visit the $\mathrm{DOI}$ to the publisher's website.

- The final author version and the galley proof are versions of the publication after peer review.

- The final published version features the final layout of the paper including the volume, issue and page numbers.

Link to publication

\section{General rights}

Copyright and moral rights for the publications made accessible in the public portal are retained by the authors and/or other copyright owners and it is a condition of accessing publications that users recognise and abide by the legal requirements associated with these rights.

- Users may download and print one copy of any publication from the public portal for the purpose of private study or research.

- You may not further distribute the material or use it for any profit-making activity or commercial gain

- You may freely distribute the URL identifying the publication in the public portal.

If the publication is distributed under the terms of Article 25fa of the Dutch Copyright Act, indicated by the "Taverne" license above, please follow below link for the End User Agreement:

www.tue.nl/taverne

Take down policy

If you believe that this document breaches copyright please contact us at:

openaccess@tue.nl

providing details and we will investigate your claim. 


\title{
Vibrational Dephasing Mechanisms in Hydrogen-Bonded Systems
}

\author{
Mischa Bonn, ${ }^{1,2}$ Marco J. P. Brugmans, ${ }^{1}$ Aart W. Kleyn, ${ }^{1}$ Rutger A. van Santen, ${ }^{2}$ and Huib J. Bakker ${ }^{1}$ \\ ${ }^{1}$ FOM-Institute for Atomic and Molecular Physics, Kruislaan 407, 1098 SJ Amsterdam, The Netherlands \\ ${ }^{2}$ Schuit Institute of Catalysis, Eindhoven University of Technology, P.O. Box 513, 5600 MB Eindhoven, The Netherlands
}

(Received 20 September 1995)

\begin{abstract}
With transient IR hole-burning spectroscopy we observe large variations in the homogeneous linewidth of the $\nu_{s}(\mathrm{O}-\mathrm{D})$ stretch vibration of differently hydrogen-bonded surface hydroxyls in zeolite catalysts. The linewidth is very sensitive to the frequency of the $\nu_{\sigma}(\mathrm{OD} \cdots X)$ hydrogen-bond vibration. By varying this frequency we obtain conclusive information on the coupling between the $\nu_{s}(\mathrm{O}-\mathrm{D})$ and the $\nu_{\sigma}(\mathrm{OD} \cdots X)$ modes.

PACS numbers: 33.20.Ea, 63.50.+x, 82.20.Rp, 78.47.+p
\end{abstract}

Vibrational phase relaxation in condensed phases has received considerable attention in recent decades because it is recognized as a powerful probe of intermolecular interaction dynamics [1]. Recent developments in timeresolved spectroscopy have allowed for, e.g., the measurement of homogeneous vibrational line shapes with IR transient hole-burning techniques [2,3] and the timeresolved observation of vibrational dephasing by means of a photon-echo experiment [4]. A great number of sophisticated models have been developed to describe vibrational line shapes [5-10], some specifically addressing the effect of hydrogen bonds [8-10]. The spectral bandwidth of a hydrogen-bonded complex is typically an order of magnitude larger than that of the unassociated molecule or oscillator, indicating that the vibrational dephasing rate is strongly influenced by hydrogen-bond formation.

The width of an absorption line can generally be described in terms of inhomogeneous and homogeneous contributions. The former are due to a static distribution of transition frequencies, whereas the latter are determined by energy $T_{1}$ and pure dephasing $T_{2}^{*}$ lifetimes of the excitation, leading to a homogeneous linewidth $2 \pi \Gamma_{\text {hom }}=$ $T_{1}^{-1}+2 T_{2}^{*-1}$. The phase correlation of an ensemble of oscillators will decay in time due to interactions of a vibration with its surroundings, since these interactions result in modulations of the $(v=0) \rightarrow(v=1)$ transition frequency. Hence the linewidth is determined by $D$, the width of the oscillator frequency distribution, and $\tau_{c}$, the time scale on which the modulation of the transition frequency occurs. For slow modulation $\left(D \tau_{c} \gg 1\right)$ the width of the absorption line is solely determined by $D$. If the modulation of the transition frequency is sufficiently rapid $\left(D \tau_{c} \lesssim 1\right)$, the net phase evolution of the oscillators will resemble that of the central frequency of the distribution. As a result decay of the macroscopic polarization will be relatively slow, resulting in a sharp absorption band. This phenomenon is well known in magnetic resonance spectroscopy as motional narrowing [11].

In this Letter we will test two theoretical models describing dephasing in hydrogen-bonded systems. The first model is based on those developed by Marechal and
Witkowski [8] and by Bratos [9], which were of a static nature (slow modulation limit, $\tau_{c} \rightarrow \infty$ ). The key point of these theories was the recognition that dephasing of the $\nu_{s}(X-\mathrm{H})$ stretch vibration occurs through coupling to the hydrogen bridge stretching mode $\nu_{\sigma}(X \mathrm{H} \cdots Y)$, directed along the same spatial coordinate as the $\nu_{s}(X-\mathrm{H})$ highfrequency mode itself; the coordinate of the $\nu_{\sigma}(X \mathrm{H} \cdots Y)$ mode $r_{\sigma}$ determines the frequency of the $\nu_{S}(X-\mathrm{H})$ mode. In the extension of this model by Robertson and Yarwood [10] the dynamics of the coupling were incorporated by introducing a stochastic modulation of the $r_{\sigma}$ coordinate superimposed on the $\nu_{\sigma}(X \mathrm{H} \cdots Y)$ vibration. This causes a frequency modulation of the high-frequency $\nu_{s}(X-\mathrm{H})$ mode through the time evolution of $r_{\sigma}$. The anharmonic coupling term $V_{\text {anh }}$ between $\nu_{s}(X-\mathrm{H})$ and $\nu_{\sigma}(X \mathrm{H} \cdots Y)$ is linear in the coordinate $r_{\sigma}(t)$ of the $\nu_{\sigma}(X \mathrm{H} \cdots Y)$ mode: $V_{\text {anh }} \propto r_{\sigma}(t)$. This description is in sharp contrast with that of the second theoretical model by Shelby and Harris [7], where the coupling depends on the coordinate of the low-frequency mode $r_{\sigma}$ to second or higher order [7] $V_{\text {anh }} \propto r_{\sigma}^{2}(t)$. In that model the modulation of the $\nu_{s}(X-\mathrm{H})$ transition frequency occurs through anharmonic coupling to a low-frequency mode, which undergoes energy exchange with the bath.

Although both theories have been successful in accounting for existing experimental data for hydrogen-bonded systems separately $[2,12]$, the models have not been comparatively tested. From previous experiments it is clear that temperature variation $[2,13]$, change in hydrogen-bond strength due to different hydrogen-bond acceptor/donor [14], change of solvent [15], and isotopic substitution [12] lead to severe complications in the interpretation of the data, since both $D$ and $\tau_{c}$ will change. We investigate the nature of the coupling between the high-frequency mode and the $\nu_{\sigma}(X \mathrm{H} \cdots Y)$ mode for differently hydrogenbonded O-D groups. We will show that a variation of exclusively the $\nu_{\sigma}(\mathrm{XH} \cdots Y)$ hydrogen-bond frequency is the decisive test for the different models. The O-D groups under investigation are hydrogen-bonded deuterated surface hydroxyl groups in zeolites. Adsorption of different adsorbates allows us to regulate $D$ and $\tau_{c}$ in a controlled manner 
through the adsorbate mass and hydrogen-bond strength. We investigate the homogeneous linewidth with IR transient spectral hole burning. Our setup consists of two independently tunable $\left(2500-4000 \mathrm{~cm}^{-1}\right.$, bandwidth $6 \mathrm{~cm}^{-1}$ at $2650 \mathrm{~cm}^{-1}$ ) $25 \mathrm{ps}$ infrared pump and probe pulses (repetition rate of $10 \mathrm{~Hz}$ ) with respective energies of $\sim 100$ and $1 \mu \mathrm{J}[16]$.

In Fig. 1, transient spectra are shown for O-D groups in the small cages of $Y$ zeolite known to be hydrogen bonded to zeolite lattice oxygen atoms [17] $\left(\mathrm{OH} \cdots \mathrm{O}_{\text {latt }}\right.$, upper panel) and O-D groups in the large cages to which gaseous nitrogen is adsorbed [18] (lower panel). Both the center frequency of the absorption band (see inset, Fig. 1) and the population lifetime $T_{1}$ of $45 \mathrm{ps}$ are the same for both O-D groups [19] (as we observed previously for the nondeuterated $\mathrm{OH} \cdots \mathrm{O}_{\text {latt }}$ and $\mathrm{OH} \cdots \mathrm{N}_{2}$ groups [20]), implying an equal hydrogen-bond strength [20, 21]. For the $\mathrm{OH} \cdots \mathrm{O}_{\text {latt }}$ groups, the pump pulse burns a hole in the absorption spectrum (see also Ref. [16]), whereas, remarkably, for the OD $\cdots \mathrm{N}_{2}$ groups the whole absorption band decreases in amplitude. We have shown previously that the hydrogen bond between the O-D

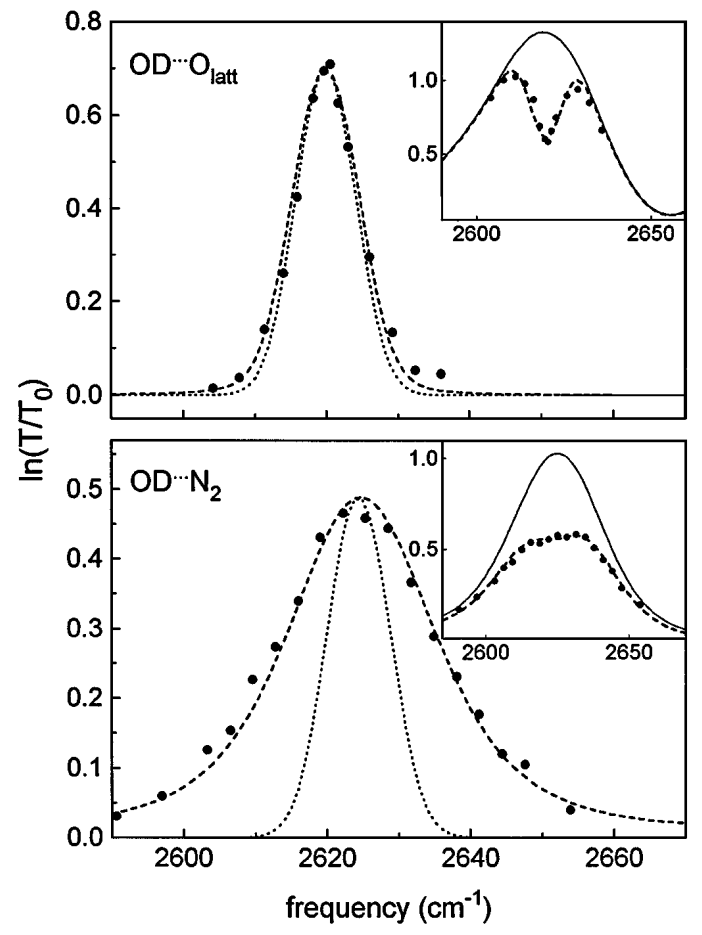

FIG. 1. Relative transmission changes due to the pump pulse tuned to the peak of the absorption band as a function of probe frequency, at 20 ps delay between pump and probe. Full circles are data, the dashed line is the instrumental function. Upper panel: The OD $\cdots \mathrm{O}_{\text {latt }}$ hydroxyls of $\mathrm{D}_{0.19} Y$ at $100 \mathrm{~K}$. The width of the burnt hole is almost completely determined by the $6 \mathrm{~cm}^{-1}$ width of the laser pulses. Lower panel: The OD $\cdots \mathrm{N}_{2}$ of $\mathrm{NaD}_{0.05} Y$ at $100 \mathrm{~K}$. Virtually the whole absorption band is bleached. Insets show the absorption spectra with (dashed curve) and without (solid curve) the presence of the pump pulse. group and the adsorbate remains intact upon vibrational relaxation [20].

From the width of the hole we derive [16] that the FWHM homogeneous linewidth (assumed Lorentzian) is $0.75 \pm 0.4 \mathrm{~cm}^{-1}$ for the OD $\cdots \mathrm{O}_{\text {latt }}$ groups at $100 \mathrm{~K}$ and $13 \pm 1.5 \mathrm{~cm}^{-1}$ for the OD $\cdots \mathrm{N}_{2}$ system (dashed lines in Fig. 1) yielding pure dephasing times $T_{2}^{*}=20 \pm 10$ and $0.82 \pm 0.1 \mathrm{ps}$, respectively. The effect of power broadening by the pump pulse is taken into account in the calculations [16]. The large difference in $T_{2}^{*}$ indicates different dephasing mechanisms for the two systems. This is confirmed by the difference in temperature dependence of $T_{2}^{*}$ for the two systems shown as an Arrhenius plot in Fig. 2, obtained from $\Gamma_{\text {hom }}$ and $T_{1}$ measured over the temperature range. For the $\mathrm{OD} \cdots \mathrm{O}_{\text {latt }}$ groups an activation energy of $200 \pm 70 \mathrm{~cm}^{-1}$ is observed, and for the $\mathrm{OD} \cdots \mathrm{N}_{2}$ groups, none.

The enormous difference in the homogeneous linewidths can be understood by considering the hydrogen bond potentials for $v_{\mathrm{OD}}=0$ and $v_{\mathrm{OD}}=1$ for the two systems schematically depicted in Fig. 3. In the presence of adsorbates, the potential is strongly anharmonic and dissociative. Furthermore, it is well known that the potentials for the ground and excited states are different [21]; the hydrogen bond is stronger for $v=1$ than for $v=0$, and hence the potential energy minimum is situated at smaller $r_{\sigma}(\mathrm{O} \cdots X$ distance $)$ for $v=1$ [21]. Hence $D$ is determined by the difference between the $v=0$ and $v=1$ potentials, i.e., by a change in the hydrogen bond [21]. In contrast, for the OD $\cdots \mathrm{O}_{\text {latt }}$ the potential energy curves are dictated by lattice parameters; the OD $\cdots \mathrm{O}_{\text {latt }}$ distance is determined not by the electrostatic interaction between the deuterium and the $\mathrm{O}_{\text {latt }}$, but by the geometry of the zeolite lattice. Hence the potential is nondissociative, and very similar for the ground and excited states of the O-D vibration. The $D$ resulting from

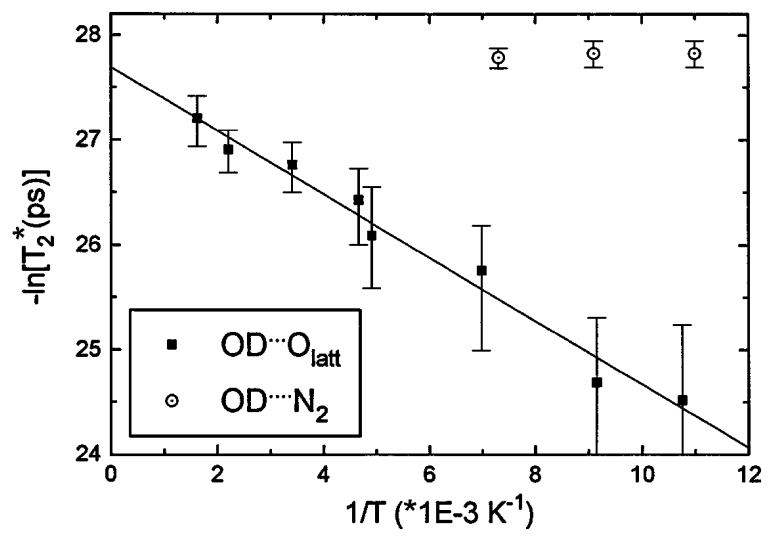

FIG. 2. Arrhenius plot of the pure dephasing time $T_{2}^{*}$ vs temperature for the OD $\cdots \mathrm{O}_{\text {latt }}$ and the OD $\cdots \mathrm{N}_{2}$ hydroxyls. For the OD $\cdots \mathrm{O}_{\text {latt }}$, a least square fit gives an activation energy of $200 \pm 70 \mathrm{~cm}^{-1}$. For the OD groups bonded to nitrogen, no such dependency is found. 

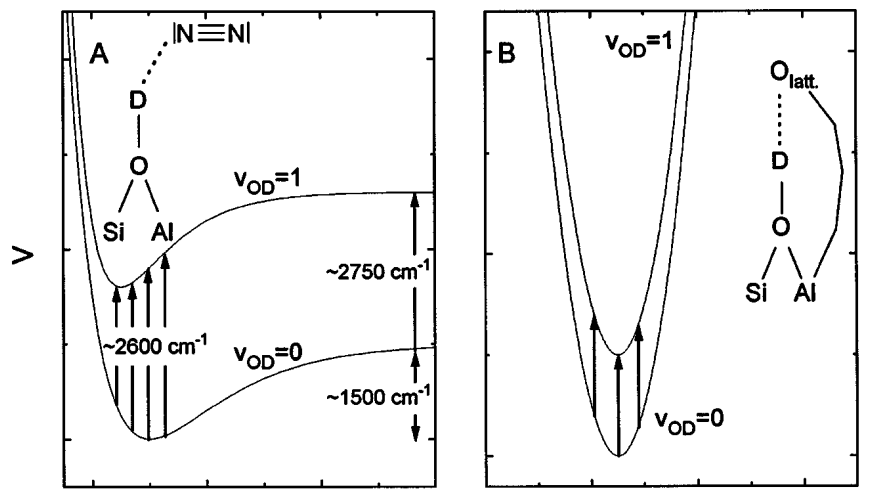

$\mathbf{r}_{\sigma}$

FIG. 3. Schematic $r_{\sigma}$ potentials for the hydrogen bonded systems: The lower curve is for the $\nu_{s}(\mathrm{O}-\mathrm{D})$ in the ground state, and the upper curve is for the $\nu_{s}(\mathrm{O}-\mathrm{D})$ in the first excited state. (a) For a hydrogen bond to an adsorbate, strongly anharmonic and dissociative. The H-bond dissociation energy is $\sim 1500 \mathrm{~cm}^{-1}$, the transition frequency $\sim 2600$ and $\sim 2750 \mathrm{~cm}^{-1}$ with and without the H-bond, respectively. After Ref. [21]. (b) For a hydrogen bond to fixed lattice oxygen atoms.

coupling to the hydrogen-bond mode will consequently be very small, and a different broadening mechanism will take over (see below). The overall inhomogeneous absorption line, in contrast, is determined by the hydrogen bond; the broadening is due to a static distribution of the hydrogen-bond strengths. This is consistent with previous experiments which showed that the vibrational lifetime $T_{1}$ increases with increasing frequency within the absorption band [22], indicating that with increasing frequency the hydrogen-bond strength decreases [17].

The observation of an activation energy, as observed for the $\left(\mathrm{OD} \cdots \mathrm{O}_{\text {latt }}\right)$ groups, is the key prediction of the Shelby-Harris model of dephasing. Exactly the same activation energy is observed for zeolite O-D groups not hydrogen bonded to the lattice. This confirms that for the $\left(\mathrm{OD} \cdots \mathrm{O}_{\text {latt }}\right)$ groups, the dephasing is not caused by the hydrogen bond, but that dephasing is due to coupling to a zeolite lattice mode. This mode is presumably the $260 \mathrm{~cm}^{-1}$ lattice oxygen mode, since there are no other modes between 100 and $300 \mathrm{~cm}^{-1}$ [23]. For the OD $\cdots \mathrm{N}_{2}$ groups where it is the hydrogen bond that is responsible for the dephasing process, the absence of a temperature dependence (neither observed for other adsorbates) does not particularly support one of the models. The absence of temperature dependence may be due to, e.g., a very small activation energy for this system (note that data points could be obtained only in the small range from 90 to $140 \mathrm{~K}$ ). Hence, the dependence of the linewidth on temperature cannot give conclusive information on the mechanism of dephasing induced by the hydrogen-bond mode.
The decisive test for the two theoretical models is to change only the frequency of the $\nu_{\sigma}$ mode: Upon an increase of the $\nu_{\sigma}$ frequency, the Shelby-Harris quadratic coupling model predicts a slower modulation of the transition frequency, since an increase of this frequency implies slower exchange with the bath. In the slow modulation limit, this will result in a narrowing of the line, since the polarization will live longer. However, the limit of slow modulation implies that vibrational population lifetimes $T_{1}$ is very short, whereas a relatively long $T_{1}$ of $45 \mathrm{ps}$ is observed. In the regime of fast modulation, appropriate to our system, the Shelby-Harris model predicts a broadening of the absorption line, since less motional narrowing will occur; due to the slower exchange $\tau_{c}$ will increase. Conversely, Robertson and Yarwood predict a narrowing of the line, since in their model the coordinate $r_{\sigma}$ determines the dephasing directly and $r_{\sigma}(t)$ will fluctuate more rapidly with increasing $\nu_{\sigma}(X \mathrm{H} \cdots Y)$ frequency. If the stochastic modulation of $r_{\sigma}$ is described by an OrnsteinUhlenbeck process [10], then the rate of frequency modulation of the $\nu_{S}(X-\mathrm{H})$ mode is determined by the frequency of the hydrogen-bond stretching mode $\nu_{\sigma}$, as well as a damping parameter $\gamma: \tau_{c}=\gamma / \nu_{\sigma}^{2}$. The frequency of the $\nu_{\sigma}$ mode can be modified simply by changing the adsorbate. Methane, for example, has a lower mass but the same adsorption energy as nitrogen, resulting only in an increase in the $\nu_{\sigma}(X \mathrm{H} \cdots Y)$ frequency. The homogeneous linewidths $\Gamma_{\exp }^{\text {hom }}$ obtained after adsorption of methane, carbon monoxide, and xenon are shown in Table I. Methane produces the same shift of the O-D stretching frequency (and a very similar absorption line) but has only half the mass of nitrogen, and hence $\nu_{\sigma}$ will decrease by a factor of $\sqrt{2}$. As shown in Table I, the homogeneous linewidth decreases significantly to $8 \mathrm{~cm}^{-1}$, conclusively validating the RobertsonYarwood model for this system. For xenon $\tau_{c}$ will be relatively large [high mass, therefore low $\nu_{\sigma}(\mathrm{OD} \cdots X)$ frequency], and we observe a homogeneous linewidth of $28 \mathrm{~cm}^{-1}$, despite the smaller adsorption energy and hence smaller $D$.

The Robertson-Yarwood expression for $\tau_{c}$ provides a quantitatively correct description of our data. Assuming a damping parameter $\gamma$ of $50 \mathrm{~cm}^{-1}$ in accordance with Refs. $[12,14,15]$, and a $\nu_{\sigma}(\mathrm{OD} \cdots X)$ frequency of $100 \mathrm{~cm}^{-1}$ for $X=\mathrm{N}_{2}$ [24], we find that a value of $46 \mathrm{~cm}^{-1}$ for $D$ is required to account for the observed hole of $14 \mathrm{~cm}^{-1}$ (implying $D \tau_{c}=0.23$, i.e., the modulation is in the intermediate regime and motional narrowing is important in the description of the linewidth). We take this set as a reference to calculate the linewidths of the other adsorbates. If the degree of anharmonicity of the $(\mathrm{OD} \cdots X)$ potential (assumed Morse) remains the same for the different adsorbates, then for the other adsorbates the $\nu_{\sigma}(\mathrm{OD} \cdots X)$ frequency is readily calculated as a function of adsorption energy $E_{\text {ads }}$ and adsorbate mass $M_{\text {ads }}$ (viz. the reduced mass of this vibration). The calculated linewidths resulting from this straightforward procedure 
TABLE I. Characteristics and effects of different adsorbates on zeolites $Y$ and Mordenite: The shift from the original O-D stretch frequency by the adsorbate $\Delta \bar{\nu}_{\mathrm{OD}}$, the width of this line $\Gamma_{\mathrm{OD}}$, the heat of adsorption $\mathrm{E}_{\mathrm{ads}}$, the experimentally observed holewidth $\Gamma_{\text {hom }}^{\mathrm{exp}}$, calculated spectral distributions $D$, calculated correlation times $\tau_{c}$, and the calculated holewidth $\Gamma_{\text {hom }}^{\mathrm{calc}}$. All widths are FWHM and errors denote $2 \sigma$

\begin{tabular}{ccccccccccc}
\hline \hline Adsorbate & Zeolite & $\begin{array}{c}M_{\text {ads }} \\
(\text { a.m.u. })\end{array}$ & $\begin{array}{c}\Delta \tilde{\nu}_{\mathrm{OD}} \\
\left(\mathrm{cm}^{-1}\right)\end{array}$ & $\begin{array}{c}\Gamma_{\mathrm{OD}} \\
\left(\mathrm{cm}^{-1}\right)\end{array}$ & $\begin{array}{c}E_{\mathrm{ads}}^{\mathrm{a}} \\
(\mathrm{eV})\end{array}$ & $\begin{array}{c}\Gamma_{\text {hom }}^{\mathrm{exp}} \\
\left(\mathrm{cm}^{-1}\right)\end{array}$ & $\begin{array}{c}D \\
\left(\mathrm{~cm}^{-1}\right)\end{array}$ & $\begin{array}{c}\tau_{c} \\
(\mathrm{~s})\end{array}$ & $\begin{array}{c}D \tau_{c} \\
\Gamma_{\text {hom }}^{\mathrm{calc}} \\
\left(\mathrm{cm}^{-1}\right)\end{array}$ \\
\hline $\mathrm{N}_{2}$ & D-Mor & 32 & $65( \pm 3)$ & $61( \pm 3)$ & 0.16 & $13( \pm 1)$ & 51 & $1.66 \times 10^{-13}$ & 0.25 & $13(\mathrm{ref})$ \\
& $\mathrm{D}-Y$ & 32 & $60( \pm 3)$ & $42( \pm 3)$ & 0.16 & $13( \pm 1)$ & 51 & $1.66 \times 10^{-13}$ & 0.25 & $13(\mathrm{ref})$. \\
$\mathrm{CH}_{4}$ & $\mathrm{D}-\mathrm{Mor}$ & 16 & $60( \pm 3)$ & $50( \pm 3)$ & 0.15 & $8( \pm 1)$ & 47.5 & $1.14 \times 10^{-13}$ & 0.16 & 7.5 \\
$\mathrm{CO}$ & $\mathrm{D}-Y$ & 32 & $168( \pm 3)$ & $65( \pm 3)$ & 0.23 & $22( \pm 2)$ & 73 & $1.12 \times 10^{-13}$ & 0.24 & 19.5 \\
$\mathrm{Xe}$ & D-Mor & 131.3 & $45( \pm 6)$ & $30( \pm 4)$ & 0.12 & $28( \pm 4)$ & 38 & $11.8 \times 10^{-13}$ & 1.34 & 29.8 \\
\hline \hline
\end{tabular}

${ }^{\mathrm{a}}$ Heats of adsorption for $\mathrm{CO}$ and $\mathrm{N}_{2}$ obtained from Ref. [25], others are estimated from $\Delta \tilde{\nu}_{\mathrm{OD}}$ (see Ref. [24]).

are shown in Table I, where $\gamma$ is assumed to be independent of adsorbate and $D$ scales with the heat of adsorption. The agreement with the data is satisfactory, and it should be noted that the results depend only weakly on the assumed reference values for $\gamma$ and $\nu_{\sigma}$ for nitrogen.

In conclusion, we have investigated the influence of hydrogen bonds on the vibrational line shape. Depending on the hydrogen-bond potential, the homogeneous linewidth in hydrogen-bonded systems is not necessarily determined by coupling to the low-frequency hydrogen-bond mode. When dephasing is caused by the hydrogen bond, in the case of the O-D groups with adsorbates, it is clear that the physical picture behind the Robertson-Yarwood model is correct: Dephasing of the $\nu_{s}(\mathrm{OD})$ mode occurs by coupling to the $\nu_{\sigma}(\mathrm{OD} \cdots X)$ mode. This coupling is linear in the hydrogen-bond coordinate $r_{\sigma}$, which is stochastically modulated in time.

This work is part of a collaborative research program of the Stichting Fundamenteel Onderzoek van de Materie (Foundation for Fundamental Research on Matter) with financial support from the Nederlandse Organisatie voor Wetenschappelijk Onderzoek (Netherlands Organization for the Advancement of Research), and the Schuit Institute of Catalysis at the Eindhoven University of Technology.

[1] D. W. Oxtoby, Adv. Chem. Phys. 40, 1 (1979).

[2] H. Graener, T. Q. Ye, and A. Laubereau, Phys. Rev. B 41, 2597 (1990).

[3] H. Graener, G. Seifert, and A. Laubereau, Phys. Rev. Lett. 66, 2092 (1991).

[4] D. Zimdars, A. Tokmakoff, S. Chen, S. R. Greenfield, and M. D. Fayer, Phys. Rev. Lett. 70, 2718 (1993).

[5] R. Kubo, J. Phys. Soc. Jpn. 9, 935 (1954).

[6] P. W. Anderson, J. Phys. Soc. Jpn. 9, 316 (1954).

[7] R. M. Shelby, C. B. Harris, and P. A. Cornelius, J. Chem. Phys. 70, 34 (1979); S. Marks, P. A. Cornelius, and C. B. Harris, J. Chem. Phys. 73, 3069 (1980).
[8] Y. Marechal and A. Witkowski, J. Chem. Phys. 48, 3697 (1968).

[9] S. Bratos, J. Chem. Phys. 63, 3499 (1975).

[10] J. Yarwood and G. N. Robertson, Nature (London) 257, 41 (1975); G. N. Robertson and J. Yarwood, Chem. Phys. 32, 267 (1978).

[11] C.P. Slichter, Principles of Magnetic Resonance (Springer-Verlag, Berlin, 1989), 3rd ed.

[12] J. Yarwood, R. Ackroyd, and G. N. Robertson, Chem. Phys. 32, 283 (1978).

[13] M. I. Cabaço, M. Besnard, and Y. Yarwood, Mol. Phys. 75, 139 (1992).

[14] B. Czarnik-Matusewicz and J. P. Hawranek, Spectrochim. Acta, Part A 43, 791 (1987).

[15] K. Tokhadze, N. Dubovna, Z. Mielke, M. WierzejewskaHnat, and H. Ratajczak, Chem. Phys. Lett. 202, 87 (1993).

[16] M.J.P. Brugmans, H.J. Bakker, and A. Lagendijk, J. Chem. Phys. (to be published).

[17] M. J. P. Brugmans, A. W. Kleyn, A. Lagendijk, W. P. J. H. Jacobs, and R. A. van Santen, Chem. Phys. Lett. 217, 117 (1994).

[18] F. Wakabayashi, J. Kondo, W. Akihide, K. Domen, and C. Hirose, J. Phys. Chem. 97, 10761 (1993).

[19] M. Bonn, M. J. P. Brugmans, H. J. Bakker, A. W. Kleyn, and R. A. van Santen (to be published).

[20] M. Bonn, M. J. P. Brugmans, A. W. Kleyn, and R. A. van Santen, Chem. Phys. Lett. 233, 309 (1995).

[21] C. Sandorfy, in Hydrogen Bonds, edited by P. Schuster (Springer-Verlag, Berlin, 1984), p. 41.

[22] M. Bonn, M.J.P. Brugmans, A.W. Kleyn, R. A. van Santen, and A. Lagendijk, Stud. Surf. Sci. Catal. 84, 493 (1994).

[23] N. D. Baker, G. A. Ozin, and J. Godber, Catal. Rev. Sci. Eng. 27, 591 (1985).

[24] D. Hadži and S. Bratos, in The Hydrogen Bond, edited by P. Schuster, G. Zundel, and C. Sandorfy (North-Holland, Amsterdam, 1976), Vol. 2, p. 565.

[25] K. M. Neyman, P. Strodel, S. P. Ruzakin, N. Schlensog, H. Knözinger, and N. Rösch, Catal. Lett. 31, 273 (1995). 